\title{
PIXEL-LEVEL SIMILARITY FUSION FOR IMAGE CLASSIFICATION
}

\author{
Alex Pappachen James
}

\author{
Queensland Micro- and nanotechnology centre, Griffith University, Australia, QLD 4111
}

\begin{abstract}
Recent research shows that local similarity calculations play a significant role in improving the recognition performance of template matching systems. We present a new scheme for parametric similarity calculation and fusion for image classification. State-of-the-art recognition results are obtained using the proposed method for a difficult task involving face images.
\end{abstract}

Index Terms - Classification, Similarity, Template matching

\section{INTRODUCTION}

The idea of similarity is widely used in the studies involving pattern matching and analysis [1-10]. In the past several decades, deeper insights into the psychological and physical mechanisms of pattern classification have reinforced the notion of similarity as important to decision making mechanism in humans [4-9].

Similarity calculations have been applied in the pattern classification schemes to determine the class of an unknown pattern or a group of patterns. Most popular are the measures of similarity that are based on correlation and grouped distances [10]. Contrary to these measures that are largely dependent on the calculation of correlation between a group of pixels, this paper presents a method to fuse similarities at pixel level. An immediate advantage with such an approach of similarity fusion over conventional approaches is that the method becomes suitable for both pixel-level features and features formed from group of pixels.

\section{PRESENTED METHOD}

A numerical similarity between two objects $x_{l}$ and $x_{t}$ can be represented as ' 1 ' when they are highly similar and ' 0 ' when they are least similar. We introduce a parametric min-max similarity $\delta_{i j}^{A}$ that can be used to achieve such numerical similarities:

$$
\delta_{i j}^{\alpha}=\left[\frac{\min \left(x_{l}(i, j), x_{t}(i, j)\right)}{\max \left(x_{l}(i, j), x_{t}(i, j)\right)}\right]^{A}
$$

The support of Queensland Micro- and nanotechnology centre is acknowledged. Acknowledgments to Prof. Kasim Al-Aubaidy for the invitation. where $A$ is an exponent parameter and $(i, j)$ is the location of the pixel (or feature) in an image. An increased value of $A$ results in stricter decision function.
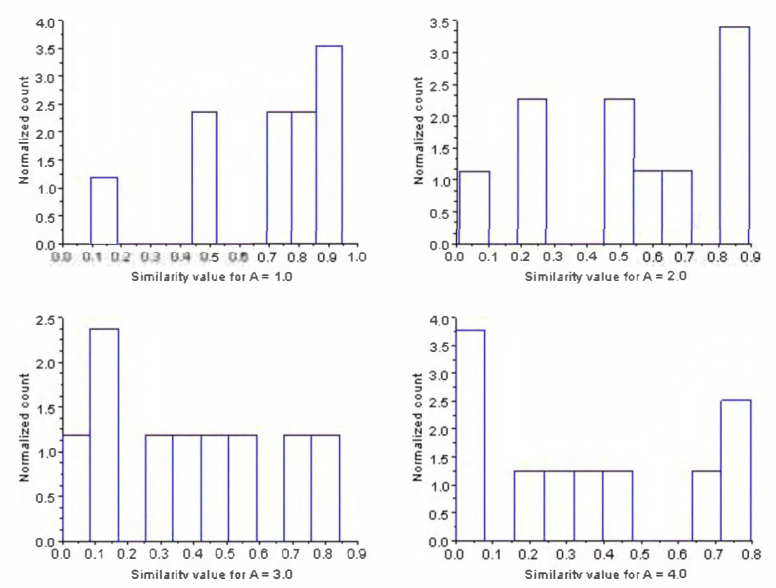

Figure 1. The histograms showing the role of ratio similarity exponent in the comparison between two randomly generated data having a uniform distribution.

Figure 1 shows the comparison between a randomly generated data with uniform distribution that is strictly bounded within a range of $[0,1]$. It can be seen that from Fig 1 , the first exponent where $A=1$, the proposed similarity function captures the most similarity. The subsequent values of $A$ reinforce only those similarities that are highly similar (close to a value of ' 1 ').

Figure 2 shows a less likely situation where the data itself is of exponential nature. The exponential data is generated by using uniformly distributed data in an exponential function having a power of 10. Clearly for the situations $A>1$, it can be seen that the proposed min-max exponential similarity provides a threshold decision by reducing the false similarities and differences.

Figure 3 shows a very likely case of gaussian data having a mean of 0.5 and standard deviation of 0.2 . This type of data is widely used in data processing applications that undergoes gaussian normalization. In such situation the similarities for low values of $A$ are distributed uniformly. We need to avoid such distribution of similarity as we want to have a clear representation of true similarity and true difference. Uniform similarity would result in ambiguous similarity and make the global decision likely to be wrong. Again with increasing the values of $A$ it becomes certain that the strong similarity values become distinguishable 

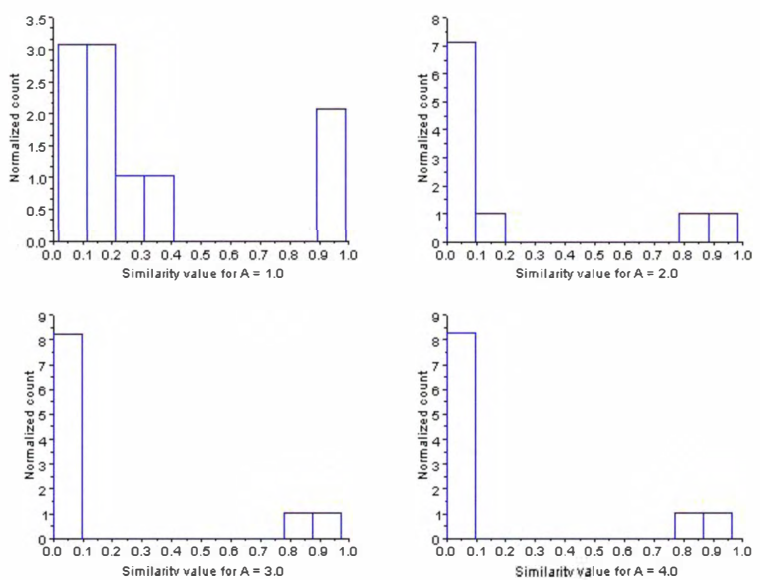

Figure 2. The histograms showing the role of ratio similarity exponent in the comparison between two randomly generated data having a exponential distribution
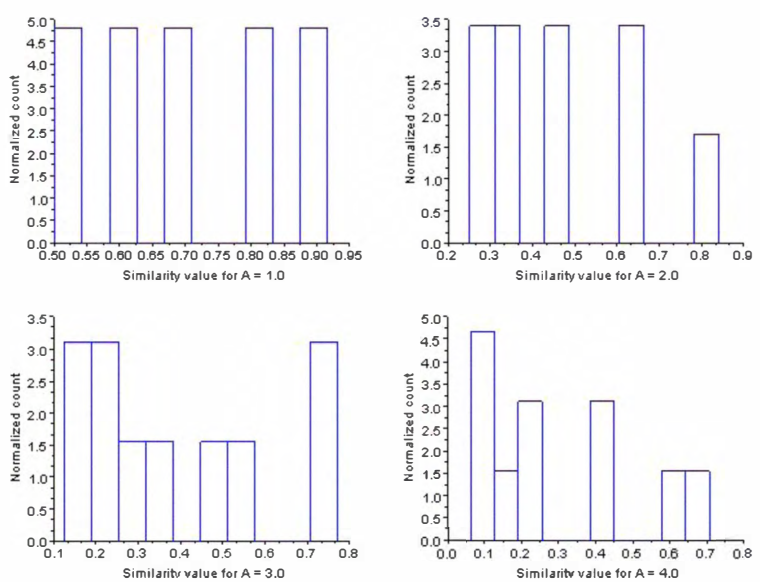

Figure 3. The histograms showing the role of ratio similarity exponent in the comparison between two randomly generated data having a gaussian distribution

from the week ones.

The local similarity $s$ between the like pixels of two comparing images are calculated using the following similarity fusion mechanism:

$$
s(i, j)=\sum_{A=1}^{N} \delta_{i j}^{A}
$$

where the parameter $N$ is determined empirically, optimum at a value 6 . The global similarity $S$ between two comparing images are then calculated as:

$$
S=\sum_{i=1}^{I} \sum_{j=1}^{J} s(i, j)
$$

In an image recognition experiment involving recognition of a test image with respect to multiple library images, similarity calculations are essential for classification of the test image. Multiple library images result in multiple similarity scores $S^{p}$, where $p$ signifies the index of the

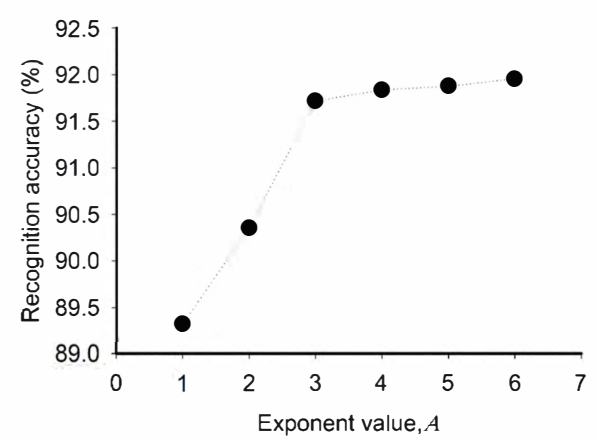

Figure 4. Graphical illustration showing the performance improvement using similarity fusion at pixel level

compared library image. The best match is determined by ranking these similarities followed by selecting the index of the top rank.

\section{EXAMPLE : FACE RECOGNITION}

AR face recognition database has been used to verify the recognition ability of the presented method [11]. The database consists of face images with various levels of natural variability. This is required to study the performance of the proposed similarity fusion in a standard template matching problem. A specific problem that has proved to be very difficult in recognition of face images is the single sample per person face recognition problem. Limitation in the number of images per person in the library results in low number of stable pixels for comparison with the test image. This makes the recognition task very difficult and often results in poor recognition accuracies.

For our experiments we use 100 images in the library with each image representing a face (a unique class). 2500 face images with varying degrees of natural variability are used for testing the recognition performance. The face images are localized and cropped to a size of $160 \times 120$ pixels based on the location of eye-coordinates. Before the presented similarity fusion method is applied, every image undergoes a preprocessing filtering (using local standard deviation filter [12]). The standard deviation filter window size is fixed at $7 \times 5$ pixels determined empirically to be optimal.

Figure 4 shows the result of the experiment performed on AR database. The application of the presented similarity fusion method at pixel level increases the recognition rate with increasing values of $A$. Further, the local similarity method outperforms global similarity method such as correlation by about $10 \%$. Figure 5 shows the histogram of pixel intensity values of gallery and test images after the application of standard deviation filter. It can be seen that application of standard deviation filter results in highly skewed exponential distribution indicative of the removal of intensity offsets in images due to changes in illumination. This also means that edges and spatial variations in the images are only represented. Figure 6 shows the dis- 


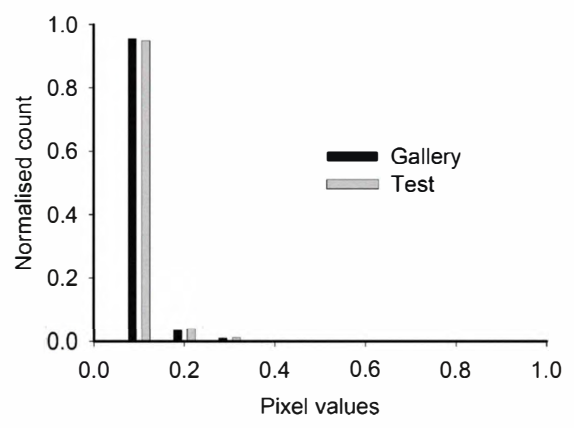

Figure 5. Histogram of pixel intensities of test and gallery image after application of standard deviation filter.

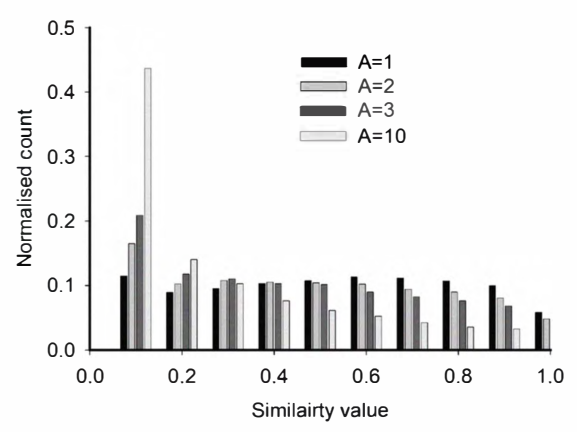

Figure 6. The histograms showing the role of ratio similarity exponent in the comparison between test and gallery images.

tribution of pixels-wise similarities for different values of A. The proposed similarity fusion method forces the decisions are made on highly skewed exponential with increased values of A. It can be seen from Fig. 6 an increased value $A$ forces an increased number of low similarity values, however an increased recognition accuracy as shown in Fig. 4. This would indicate the ability of the method to remove false similarities. As a result, the class discrimination between inter-class similarity calculations in improved.

\section{CONCLUSION}

This paper presented a novel method to fuse the similarity between compared images at pixel level. The method is applicable for wide range of data types and is useful in practical recognition problems such as shown through the example of face recognition.

It was shown that the use of increased number of similarities through the fusion parameter $A$ results in increased classification accuracy. The increased class discriminatory ability is attributed to its ability to differentiate between false and true similarities with increased values of $A$.

An extended application of such a method can be in multi-biometric and multi-sensor systems, where data with different distributions need to be compared and similarities calculated.

\section{REFERENCES}

[1] E. Xing, A. Ng, M. Jordan, and S. Russell, "Distance metric learning with application to clustering with side-information," Advances in Neural Information Processing Systems, vol. 15, pp. 505-512, 2002.

[2] A. Tversky, "Features of similarity," Psychological Review, vol. 84, pp. 327-352, 1977.

[3] L. L. Thurstone, "A law of comparative judgment," Psychological Review, vol 34, pp. 273-286, 1927.

[4] W. S. Torgerson, " Multidimensional scaling: I. Theory and method", Psychometrika, vol. 17, pp. 401419, 1952.

[5] G.-D. Guo, A. K. Jain, W.-Y. Ma, and H.-J. Zhang, "Learning similarity measure for natural image retrieval with relevance feedback", IEEE Transactions on Neural Networks, vol. 13, pp. 811-820, 2002.

[6] D. W. Hosmer, and S. Lemeshow, Applied Logistic Regression, 2nd ed. New York, NY: Wiley.

[7] W. James, Principles of psychology, New York: Holt, 1890

[8] C. L. Krumhansl, "Concerning the applicability of geometric models to similarity data: The interrelationship between similarity and spatial density", Psychological Review, vol. 85, PP. 445-463, 1978.

[9] R. N. Shepard, "Toward a universal law of generalization for psychological science", Science, vol. 237, pp. 1317-1323, 1987.

[10] R. Brunelli, Template Matching Techniques in Computer Vision: Theory and Practice, Wiley, 2009.

[11] A.M. Martinez, and R. Benavente, "The AR Face Database", CVC Technical Report 24, 1998.

[12] A. P. James, and S. Dimitrijev, "Face Recognition Using Local Binary Decisions", IEEE Signal Processing Letters, vol. 15, no. 1, pp. 821-824, 2008. 